CERN-PH-TH/2004-185

\title{
Magnetized birefringence and CMB polarization
}

\author{
Massimo Giovannini \\ Department of Physics, Theory Division, CERN, 1211 Geneva 23, Switzerland
}

\begin{abstract}
The polarization plane of the cosmic microwave background radiation can be rotated either in a magnetized plasma or in the presence of a quintessential background with pseudoscalar coupling to electromagnetism. A unified treatment of these two phenomena is presented for cold and warm electron-ion plasmas at the pre-recombination epoch. The electron temperature is only relevant to the relativistic correction of the cold plasma results. The spectrum of plasma excitations is obtained from a generalized Appleton-Hartree equation, describing simultaneously the high-frequency propagation of electromagnetic waves in a magnetized plasma with a dynamical quintessence field. It is shown that these two effects are comparable for the plausible range of parameters allowed by present constraints. It is then argued that the generalized expressions derived in the present study may be relevant for direct searches of a possible rotation of the cosmic microwave background polarization.
\end{abstract}


If linearly polarized radiation passes through a cold (or warm) plasma containing a magnetic field, the polarization plane of the wave can be rotated since the two circular polarizations (forming the linearly polarized beam) are travelling at different speeds. This effect has been studied in a variety of different (but related) frameworks even in relativistic QED plasmas and in the presence of extra-dimensions (see, for instance, [1]).

The cosmic microwave background (CMB) has a weak degree of linear polarization, which is the direct result of Thompson scattering. If CMB is linearly polarized, then its polarization plane can be rotated provided a sufficiently strong magnetic field is present around the time of decoupling [2]. Typical values of magnetic fields, compatible with other astrophysical constraints, are of the order of $B_{0} \lesssim 10^{-4} \mathrm{G}$ at the decoupling time (see, for instance, [3]).

An apparently unrelated possibility is that the quintessence field $\sigma$ has pseudoscalar couplings to electromagnetism [4]. Couplings to ordinary matter, even if suppressed by the Planck scale, may lead to observable long-range forces and time dependence of the constants of nature. An approximate global symmetry may suppress these couplings even further as argued in [5] (second reference). This possibility may also imply a rotation of the plane of polarized emission, and radio-astronomical implications of this type of cosmic birefringence were investigated through various steps [5]. Typical values of the mass of $\sigma$ are of the order of $10^{-33} \mathrm{eV}$ in such a way that, between redshifts 0 and $3, \sigma$ can start dominating the background with energy density $m^{2} \sigma_{0}^{2} \sim \Lambda^{4}$, where $\Lambda \simeq 10^{-3} \mathrm{eV}$ and $\sigma_{0} \simeq M_{\mathrm{P}} \sim 10^{18} \mathrm{GeV}$. The effect of parity violating interactions on $\mathrm{CMB}$ polarization has been recently discused in [6].

In this paper a unified discussion of Faraday rotation and cosmic birefringence will be presented for the specific case of the pre-recombination plasma. The possible interference of these two effects will be scrutinized in both the cold and warm plasma approximations. A related aspect of the present analysis will be to study the range of validity of the Faraday rotation estimates.

Let us start by discussing the typical scales involved in the problem. Right before decoupling, the temperature of the plasma is of the order of $0.3 \mathrm{eV}$ and the typical free electron density can be estimated as

$$
n_{e} \simeq x_{\mathrm{e}}\left(\Omega_{\mathrm{b}} h_{0}^{2}\right)(1+z)^{3} \times 10^{-5} \mathrm{~cm}^{-3},
$$

where $x_{e}$ is the ionization fraction, $h_{0}$ is the indetermination associated with the Hubble constant, $\Omega_{b}$ is the fraction of critical density in baryons, and $z$ is the redshift. For typical values of the parameters the electron density is of the order of $n_{\mathrm{e}} \sim 10^{3} \mathrm{~cm}^{-3}$.

Right before decoupling, the plasma is globally neutral and the ion density equals the electron density, i.e. $n_{\mathrm{i}} \simeq n_{\mathrm{e}}=n_{0}$, denoting with $n_{0}$ the common electron-ion number density. The global neutrality of the plasma occurs for typical length scales $L \gg \lambda_{\mathrm{D}}$ where

$$
\lambda_{\mathrm{D}}=\sqrt{\frac{T_{\mathrm{ei}}}{8 \pi e^{2} n_{0}}} \simeq 10\left(\frac{n_{0}}{10^{3} \mathrm{~cm}^{-3}}\right)^{-1 / 2}\left(\frac{T_{\mathrm{ei}}}{0.3 \mathrm{eV}}\right)^{1 / 2} \mathrm{~cm} .
$$


is the Debye screening length whose value is much smaller, for the same choice of physical parameters, than the electron and photon mean free paths, i.e. $\ell_{\mathrm{e}} \simeq 5.7 \times 10^{7} \mathrm{~cm}$ and $\ell_{\gamma} \simeq 10^{4}(1+z)^{-2}\left(\Omega_{\mathrm{b}} h_{0}^{2}\right)^{-1} \mathrm{Mpc}$.

If the plasma is not magnetized, the only relevant frequency scales of the problem are the plasma frequencies, which can be constructed from the electron and ion densities, i.e.

$$
\omega_{\mathrm{pe}}=\sqrt{\frac{4 \pi e^{2} n_{0}}{m_{\mathrm{e}}}} \simeq 2\left(\frac{n_{e}}{10^{3} \mathrm{~cm}^{-3}}\right)^{1 / 2} \mathrm{MHz}, \quad \omega_{\mathrm{pi}}=\sqrt{\frac{4 \pi e^{2} n_{0}}{m_{\mathrm{i}}}} \simeq 40\left(\frac{n_{e}}{10^{3} \mathrm{~cm}^{-3}}\right)^{1 / 2} \mathrm{kHz},
$$

where, for practical estimates, the numerical value $m_{\mathrm{i}} \simeq m_{\mathrm{p}}=0.94 \mathrm{GeV}$ has been assumed.

The frequencies given in Eq. (3) enter the dispersion relations, determining the group velocity of an electromagnetic signal in the plasma. The plasma frequencies for both electrons and ions are much larger than the collision frequencies constructed from the inverse of the mean free paths, i.e. $\omega_{\text {ce }} \sim 520 \mathrm{~Hz}$ and $\omega_{\text {ci }} \sim 2.4 \mathrm{~Hz}$. The plasma can then be described, to a very good approximation, within a two-fluid framework $[7,8]$.

If the plasma is magnetized, two new frequency scales arise in the problem, namely the electron and ion gyrofrequencies, i.e.

$$
\omega_{\mathrm{Be}}=\frac{e B_{0}}{m_{\mathrm{e}} c} \simeq 18.08\left(\frac{B_{0}}{10^{-3} \mathrm{G}}\right) \mathrm{kHz}, \quad \omega_{\mathrm{Bi}}=\frac{e B_{0}}{m_{\mathrm{i}} c} \simeq 9.66\left(\frac{B_{0}}{10^{-3} \mathrm{G}}\right) \mathrm{Hz},
$$

where $B_{0}$ is the magnetic field strength at the corresponding epoch.

The electron and ion gyrofrequencies, together with the plasma frequencies of Eq. (3), affect the dispersion relations in the case of a magnetized plasma. The maximum of the CMB can be determined, from the Wien law, around $1.7 \mathrm{GHz}$; we will then be interested in the frequency range between, say, 1 and $100 \mathrm{GHz}$. In this frequency range $\omega_{\mathrm{CMB}} \gg \omega_{\mathrm{pe}}>\omega_{\mathrm{Be}}$. Since $\omega_{\mathrm{pe}}>\omega_{\mathrm{pi}}$ and $\omega_{\mathrm{Be}}>\omega_{\mathrm{Bi}}$, we will also have $\omega_{\mathrm{CMB}} \gg \omega_{\mathrm{pi}}>\omega_{\mathrm{Bi}}$ for the fiducial set of parameters introduced so far.

Defining the appropriately rescaled electron and ion densities, $n_{\mathrm{e}}=a^{3} \tilde{n}_{\mathrm{e}}$ and $n_{\mathrm{i}}=a^{3} \tilde{n}_{\mathrm{i}}$ in a conformally flat geometry of Friedmann-Robertson-Walker background characterized by a scale factor $a(\eta)$ and by the line element $d s^{2}=a^{2}(\eta)\left[d \eta^{2}-d \vec{x}^{2}\right]$, the continuity equations for the charge densities reads

$$
\begin{aligned}
& n_{\mathrm{e}}^{\prime}+3 w_{\mathrm{e}} \mathcal{H} n_{\mathrm{e}}+\left(w_{\mathrm{e}}+1\right) \vec{\nabla} \cdot\left(n_{\mathrm{e}} \overrightarrow{\mathrm{v}}_{\mathrm{e}}\right)=0, \\
& n_{\mathrm{i}}^{\prime}+3 w_{\mathrm{i}} \mathcal{H} n_{\mathrm{i}}+\left(w_{\mathrm{i}}+1\right) \vec{\nabla} \cdot\left(n_{\mathrm{i}} \vec{v}_{\mathrm{i}}\right)=0,
\end{aligned}
$$

where $\mathcal{H}=a^{\prime} / a$; the prime denotes a derivation with respect to the comformal time coordinate $\eta ; w$ is the barotropic index for the electron or ion fluid.

Both electrons and ions are non-relativistic before decoupling. Hence the barotropic index $w$ will be close to zero to a good approximation. For instance, the energy and pressure densities of an ideal electronic gas are given by

$$
\rho_{\mathrm{e}}=n_{\mathrm{e}}\left(m_{\mathrm{e}}+\frac{3}{2} T_{\mathrm{e}}\right), \quad p_{\mathrm{e}}=n_{\mathrm{e}} T_{\mathrm{e}}
$$


and since $w_{\mathrm{e}, \mathrm{i}}=T_{\mathrm{e}, \mathrm{i}} / m_{\mathrm{e}, \mathrm{i}}, w_{\mathrm{e}, \mathrm{i}} \ll 1$ as far as $T_{\mathrm{e}, \mathrm{i}} \ll m_{\text {,ie }}$.

In the cold plasma approximation the temperature of the ions and of the electrons vanishes. In the warm plasma approximation the temperature of the two charged species may be very small but non-vanishing. The warm plasma treatment will lead, in practice, only to an effective correction of the plasma frequency. Since the cold plasma results turn out to be, a posteriori, rather accurate, the discussion will be presented in terms of the cold plasma description, while the results of the warm plasma treatment will be stated later.

To have a self-consistent set of two-fluid equations, Eqs. (5) and (6) will be supplemented by the evolution equations of the velocity fields and of the electromagnetic field, namely

$$
\begin{aligned}
& \rho_{\mathrm{e}}\left[\vec{v}_{\mathrm{e}}^{\prime}+\mathcal{H} \vec{v}_{\mathrm{e}}+\left(\vec{v}_{\mathrm{e}} \cdot \vec{\nabla}\right) \vec{v}_{\mathrm{e}}\right]=-n_{\mathrm{e}} e\left(\vec{E}+\frac{\vec{v}_{\mathrm{e}}}{c} \times \vec{B}\right), \\
& \rho_{\mathrm{i}}\left[\vec{v}_{\mathrm{i}}^{\prime}+\mathcal{H} \vec{v}_{\mathrm{i}}+\left(\vec{v}_{\mathrm{i}} \cdot \vec{\nabla}\right) \vec{v}_{\mathrm{i}}\right]=n_{\mathrm{i}} e\left(\vec{E}+\frac{\vec{v}_{\mathrm{i}}}{c} \times \vec{B}\right),
\end{aligned}
$$

where $\vec{E}=a^{2} \overrightarrow{\mathcal{E}}$ and $\vec{B}=a^{2} \overrightarrow{\mathcal{B}}$ are the conformally rescaled electromagnetic fields obeying the following set of generalized Maxwell equations:

$$
\begin{aligned}
& \vec{\nabla} \cdot \vec{E}=4 \pi e\left(n_{\mathrm{i}}-n_{\mathrm{e}}\right)+\frac{\beta}{M} \vec{\nabla} \sigma \cdot \vec{B}, \\
& \vec{\nabla} \cdot \vec{B}=0, \quad \vec{\nabla} \times \vec{E}=-\frac{1}{c} \vec{B}^{\prime} \\
& \vec{\nabla} \times \vec{B}=\frac{1}{c} \vec{E}^{\prime}-\frac{\beta}{M}\left[\sigma^{\prime} \vec{B}+\vec{\nabla} \sigma \times \vec{E}\right]+\frac{4 \pi e}{c}\left(n_{\mathrm{i}} \vec{v}_{\mathrm{i}}-n_{\mathrm{e}} \vec{v}_{e}\right),
\end{aligned}
$$

where the coupling of the electromagnetic field to the quintesssence field $\sigma$ can be derived from the action

$$
S_{\sigma}=\int d^{4} x \sqrt{-g}\left[\frac{1}{2} g^{\alpha \beta} \partial_{\alpha} \sigma \partial_{\beta} \sigma-W(\sigma)+\frac{\beta}{4 M} F_{\alpha \beta} \tilde{F}^{\alpha \beta}\right]
$$

where $F_{\alpha \beta}$ and $\tilde{F}^{\alpha \beta}$ are the Maxwell field strength and its dual; $g_{\alpha \beta}$ is the metric tensor. In the limit $\sigma \rightarrow 0$, Eqs. (10)-(12) are the usual curved-space two-fluid equations [3]. The evolution of the quintessence field will obey the homogeneous equation

$$
\ddot{\sigma}+3 H \dot{\sigma}+\frac{\partial W}{\partial \sigma}=0
$$

where the overdot denotes a derivation with respect to the cosmic time coordinate whose differential is related to the conformal time coordinate as $d t=a(\eta) d \eta$; it will be assumed that $2 W(\sigma)=m^{2} \sigma^{2}$. From Eqs. (10)-(12), the presence of the quintessence field introduces a further frequency scale into the problem, namely $\omega_{\sigma}=c(\beta / M) \sigma^{\prime}$. If the quintessence field dominates today (or between redshifts 0 and 3), $\sigma\left(t_{0}\right) \sim \Lambda^{2} / m$ where, typically, $\Lambda \sim 10^{-3} \mathrm{eV}$, and $m \sim 10^{-33} \mathrm{eV}$. The value of $\dot{\sigma}$ can be estimated, today, from Eq. (14) and it is 
$\dot{\sigma}\left(t_{0}\right) \simeq m^{2} / H_{0}$. Hence, recalling that prior to quintessential dominance, $\dot{\sigma}$ scales as $a^{-3}$, from the previous expressions

$$
\dot{\sigma}_{\mathrm{dec}} \simeq\left(\frac{H_{\mathrm{dec}}}{H_{0}}\right)^{2} \frac{m \Lambda^{2}}{H_{0}}, \quad \omega_{\sigma}=\beta\left(\frac{M}{M_{\mathrm{P}}}\right) \times 10^{-6} \mathrm{~Hz},
$$

where $H_{\mathrm{dec}} \sim 10^{6} H_{0}$; the values of $\Lambda$ and $m$ are the ones discussed above with $M \simeq M_{\mathrm{P}}$. The value of $\beta$ is rather uncertain and a conservative limit from radio-astronomical analyses would imply $\beta \lesssim 10^{-3}$ for $0 \lesssim z \lesssim 1[5]$. Notice that the action (13) can also be relevant in a class of baryogenesis models [9] (see also [10]). Similar actions also arise in the framework of theories with varying coupling constants (see for instance [11]).

Consider the combined effect of the homogeneous quintessence field (i.e. $\vec{\nabla} \sigma=0$ ) and of a background magnetic field on the spectrum of plasma excitations. Equations (5) and (6) together with Equations (8) and (9) and (10)-(12) can then be linearized in the presence of the weak background magnetic field $B_{0}$, i.e.

$$
\begin{array}{ll}
n_{\mathrm{e}, \mathrm{i}}(\eta, \vec{x})=n_{0}+\delta n_{\mathrm{e}, \mathrm{i}}(\eta, \vec{x}), & \vec{B}(\eta, \vec{x})=\vec{B}_{0}+\delta \vec{B}(\eta, \vec{x}), \\
\vec{v}_{\mathrm{e}, \mathrm{i}}(\eta, \vec{x})=\delta \vec{v}_{\mathrm{e}, \mathrm{i}}(\eta, \vec{x}), & \vec{E}(\eta, \vec{x})=\delta \vec{E}(\eta, \vec{x}) .
\end{array}
$$

Using Eq. (16), the system of Eqs. (5)-(9) and Eqs. (10)-(12) can be written as

$$
\begin{aligned}
& \delta n_{\mathrm{e}}^{\prime}+n_{0} \vec{\nabla} \cdot \delta \vec{v}_{\mathrm{e}}=0, \quad \delta n_{\mathrm{i}}^{\prime}+n_{0} \vec{\nabla} \cdot \delta \vec{v}_{\mathrm{i}}=0, \\
& \delta \vec{v}_{\mathrm{e}}^{\prime}+\mathcal{H} \delta \overrightarrow{\mathrm{v}}_{\mathrm{e}}=-\frac{e}{m_{\mathrm{e}}}\left[\delta \vec{E}+\frac{\delta \vec{v}_{\mathrm{e}}}{c} \times \vec{B}_{0}\right], \quad \delta \vec{v}_{\mathrm{i}}^{\prime}+\mathcal{H} \delta \vec{v}_{\mathrm{i}}=\frac{e}{m_{\mathrm{i}}}\left[\delta \vec{E}+\frac{\delta \vec{v}_{\mathrm{i}}}{c} \times \vec{B}_{0}\right], \\
& \vec{\nabla} \times \delta \vec{E}=-\frac{1}{c} \delta \vec{B}^{\prime}, \quad \vec{\nabla} \cdot \delta \vec{E}=4 \pi e\left(\delta n_{\mathrm{i}}-\delta n_{\mathrm{e}}\right), \\
& \vec{\nabla} \times \delta \vec{B}=\frac{1}{c} \delta \vec{E}^{\prime}-\frac{\beta}{M} \sigma^{\prime} \delta \vec{B}+\frac{4 \pi e n_{0}}{e}\left(\delta \vec{v}_{\mathrm{i}}-\delta \vec{v}_{\mathrm{e}}\right) .
\end{aligned}
$$

From Eqs. (17)-(19) the relevant dispersion relations and the associated refraction indices can be obtained by treating separately the motions parallel and perpendicular to the magnetic field direction. Defining the current direction parallel to the magnetic field as $\vec{j}_{\|}=n_{0} e\left(\delta \vec{v}_{\mathrm{i}, \|}-\delta \vec{v}_{\mathrm{e}, \|}\right)$, Eqs. (18) imply

$$
\vec{j}_{\|}^{\prime}+\mathcal{H} \vec{j}_{\|}=\frac{1}{4 \pi}\left(\omega_{\mathrm{p}, \mathrm{i}}^{2}+\omega_{\mathrm{e}, \mathrm{i}}^{2}\right) \delta \vec{E}_{\|} .
$$

Since the variation of the geometry is slow in with respect to the typical frequencies of plasma oscillations, the following adiabatic expansions can be used:

$$
\vec{j}_{\|}(\eta, \vec{x})=\vec{j}_{\|, \omega}(\vec{x}) e^{-i \int^{\eta} d \eta^{\prime} \omega\left(\eta^{\prime}\right)}, \quad \delta \vec{E}_{\|}(\eta, \vec{x})=\delta \vec{E}_{\|, \omega}(\vec{x}) e^{-i \int^{\eta} d \eta^{\prime} \omega\left(\eta^{\prime}\right)} .
$$

Thus, defining $\alpha=i \mathcal{H} / \omega \ll 1$, Eq. (21) implies that

$$
\vec{j}_{\|, \omega}=\frac{i}{4 \pi} \frac{\omega_{\mathrm{p}, \mathrm{i}}^{2}+\omega_{\mathrm{p}, \mathrm{e}}^{2}}{\omega(1+\alpha)} \delta \vec{E}_{\|, \omega}
$$


Inserting Eq. (23) into the parallel component of Eq. (20), the following equation can be obtained:

$$
\left(\vec{\nabla} \times \delta \vec{B}_{\omega}\right)_{\|}=-i \frac{\omega}{c} \epsilon_{\|}(\omega, \alpha) \delta \vec{E}_{\|, \omega}-\frac{\beta}{M} \sigma^{\prime} \delta \vec{B}_{\|, \omega},
$$

where the parallel dielectric constant is

$$
\epsilon_{\|}(\omega, \alpha)=1-\frac{\omega_{\mathrm{p}, \mathrm{i}}^{2}}{\omega^{2}(1+\alpha)}-\frac{\omega_{\mathrm{p}, \mathrm{e}}^{2}}{\omega^{2}(1+\alpha)} .
$$

Notice that only the leading curved-space correction has been kept.

With a similar procedure, also the motion in the plane orthogonal to the magnetic field direction can be solved and the evolution equations of the electric and magnetic fluctuations can then be written, in compact notation, as

$$
\begin{aligned}
& \vec{\nabla} \times \delta \vec{E}_{\omega}=i \frac{\omega}{c} \delta \vec{B} \\
& \vec{\nabla} \times \delta \vec{B}_{\omega}=-i \frac{\omega}{c} \bar{\epsilon}(\alpha, \omega) \delta \vec{E}_{\omega}-\frac{\beta}{M} \sigma^{\prime} \delta \vec{B}_{\omega}
\end{aligned}
$$

where $\delta \vec{E}_{\omega}$ and $\delta \vec{B}_{\omega}$ have to be understood as column matrices containing, in each row, the components of the electric and magnetic fields in each of the three spatial directions, while $\bar{\epsilon}(\omega, \alpha)$ is a $3 \times 3$ matrix given by

$$
\bar{\epsilon}(\omega, \alpha)=\left(\begin{array}{ccc}
\epsilon_{1}(\omega, \alpha) & i \epsilon_{2}(\omega, \alpha) & 0 \\
-i \epsilon_{2}(\omega, \alpha) & \epsilon_{1}(\omega, \alpha) & 0 \\
0 & 0 & \epsilon_{\|}(\omega, \alpha)
\end{array}\right)
$$

where $\epsilon_{\|}(\omega, \alpha)$ is defined by Eq. $(25) ; \epsilon_{1,2}(\omega, \alpha)$ are instead

$$
\begin{aligned}
& \epsilon_{1}(\omega, \alpha)=1-\frac{\omega_{\mathrm{pi}}^{2}(\alpha+1)}{\omega^{2}(\alpha+1)^{2}-\omega_{\mathrm{Bi}}^{2}}-\frac{\omega_{\mathrm{pe}}^{2}(\alpha+1)}{\omega^{2}(\alpha+1)^{2}-\omega_{\mathrm{B} \mathrm{e}}^{2}}, \\
& \epsilon_{2}(\omega, \alpha)=\frac{\omega_{\mathrm{B} \mathrm{e}}}{\omega} \frac{\omega_{\mathrm{pe}}^{2}}{\omega^{2}(\alpha+1)^{2}-\omega_{\mathrm{Be}}^{2}}-\frac{\omega_{\mathrm{Bi}}}{\omega} \frac{\omega_{\mathrm{pi}}^{2}}{\omega^{2}(\alpha+1)^{2}-\omega_{\mathrm{Bi}}^{2}} .
\end{aligned}
$$

The coordinate system can be fixed by setting $k_{x}=0$ and $k_{y}=k \sin \theta, k_{z}=k \cos \theta$ with $\vec{B}_{0}$ oriented along the $\hat{z}$ direction. Since Eqs. (26) and (27) imply

$$
\vec{\nabla} \times \vec{\nabla} \times \delta \vec{B}_{\omega}=\frac{\omega^{2}}{c^{2}} \bar{\epsilon}(\omega, \alpha) \delta \vec{B}_{\omega}-\frac{\beta}{M} \sigma^{\prime} \vec{\nabla} \times \delta \vec{B}_{\omega}
$$

the Fourier transform of Eq. (31), in the coordinate system selected previously, leads to the generalized Appleton-Hartree equation:

$$
\mathcal{A} \delta \vec{B}_{\vec{k}, \omega}=\left(\begin{array}{ccc}
{\left[1-\frac{\epsilon_{1}}{n^{2}}\right]} & -i\left[\frac{\epsilon_{2}}{n^{2}}+\frac{\omega_{\sigma}}{n \omega} c(\theta)\right] & i \frac{\omega_{\sigma}}{n \omega} s(\theta) \\
i\left[\frac{\epsilon_{2}}{n^{2}}+\frac{\omega_{\sigma}}{n \omega} c(\theta)\right] & {\left[c^{2}(\theta)-\frac{\epsilon_{1}}{n^{2}}\right]} & -s(\theta) c(\theta) \\
-i \frac{\omega_{\sigma}}{n \omega} s(\theta) & -s(\theta) c(\theta) & {\left[s^{2}(\theta)-\frac{\epsilon_{\|}(\omega, \alpha)}{n^{2}}\right]}
\end{array}\right)\left(\begin{array}{l}
\delta B_{k, \omega, x} \\
\delta B_{k, \omega, y} \\
\delta B_{k, \omega, z}
\end{array}\right)=0
$$


where the refraction index $n=c / v$ has been introduced so as to eliminate the comoving momentum, in such a way that $k=\omega / v=n \omega / c$; we have written $c(\theta)=\cos \theta$ and $s(\theta)=$ $\sin \theta$. From Eq. (32), $\mathcal{A}^{\dagger}=\mathcal{A}$, where the dagger denotes the transposed and complex conjugate of a given matrix.

The non-trivial solutions of the system of algebraic (homogeneous) equations given by formula (32) comes from setting the determinant of the coefficients equal to zero, i.e. $\operatorname{det} \mathcal{A}=$ 0 . After some algebra it is found that the determinant vanishes if

$$
\begin{aligned}
& s^{2}(\theta)\left\{\left(\frac{1}{\epsilon_{\|}}-\frac{1}{n^{2}}\right)\left[\frac{1}{n^{2}}-\frac{1}{2}\left(\frac{1}{\epsilon_{\mathrm{L}}}+\frac{1}{\epsilon_{\mathrm{R}}}\right)\right]+\frac{\omega_{\sigma}^{2}}{2 n^{2} \omega^{2}}\left(\frac{1}{\epsilon_{\mathrm{L}}}+\frac{1}{\epsilon_{\mathrm{R}}}\right)\right. \\
& -c^{2}(\theta)\left[\left(\frac{1}{n^{2}}-\frac{1}{\epsilon_{\mathrm{L}}}\right)\left(\frac{1}{n^{2}}-\frac{1}{\epsilon_{\mathrm{R}}}\right)-\frac{\omega_{\sigma}^{2}}{n^{2} \omega^{2} \epsilon_{\mathrm{R}} \epsilon_{\mathrm{R}}}\right]+\frac{\omega_{\sigma}}{n^{3} \omega}\left(\frac{1}{\epsilon_{\mathrm{L}}}-\frac{1}{\epsilon_{\mathrm{R}}}\right) c(\theta)=0,
\end{aligned}
$$

where the right-handed and left-handed dielectric constants have been defined as

$$
\begin{aligned}
& \epsilon_{\mathrm{R}}=\epsilon_{1}+\epsilon_{2}=1-\frac{\omega_{\mathrm{pi}}^{2}}{\omega\left[\omega(\alpha+1)-\omega_{\mathrm{Bi}}\right]}-\frac{\omega_{\mathrm{pe}}^{2}}{\omega\left[\omega(\alpha+1)+\omega_{\mathrm{Be}}\right]}, \\
& \epsilon_{\mathrm{L}}=\epsilon_{1}-\epsilon_{2}=1-\frac{\omega_{\mathrm{pe}}^{2}}{\omega\left[\omega(\alpha+1)-\omega_{\mathrm{Be}}\right]}-\frac{\omega_{\mathrm{pi}}^{2}}{\omega\left[\omega(\alpha+1)+\omega_{\mathrm{Bi}}\right]} .
\end{aligned}
$$

If $\omega_{\sigma}=0$, then Eq. (33) reduces exactly to the Appleton-Hartree equation known from two-fluid plasma theory [7], with the minor difference that the leading dependence upon the background geometry appears in $\epsilon_{\mathrm{R}, \mathrm{L}}$ through the function $\alpha$. The dispersion relations for a wave propagating parallel and perpendicular to the magnetic field direction can be obtained by setting, respectively, $\theta=0$ and $\theta=\pi / 2$ in Eq. (33). Consequently, the relevant equations determining the refraction index are, in this case

$$
\begin{array}{ll}
\left(n^{2}-\epsilon_{\mathrm{R}}\right)\left(n^{2}-\epsilon_{\mathrm{L}}\right)=0, & \theta=0, \\
\left(n^{2}-\epsilon_{\|}\right)\left[n^{2}\left(\epsilon_{\mathrm{L}}+\epsilon_{\mathrm{R}}\right)-2 \epsilon_{\mathrm{L}} \epsilon_{\mathrm{R}}\right]=0, & \theta=\frac{\pi}{2} .
\end{array}
$$

Equation (36) gives the usual dispersion relations for the two circular polarizations of the electromagnetic wave, i.e. $n^{2}=\epsilon_{\mathrm{R}}$ and $n^{2}=\epsilon_{\mathrm{L}}$, while Eq. (37) gives those for the "ordinary" (i.e. $n^{2}=\epsilon_{\|}$) and "extraordinary" (i.e. $n^{2}=2 \epsilon_{\mathrm{R}} \epsilon_{\mathrm{L}} /\left(\epsilon_{\mathrm{R}}+\epsilon_{\mathrm{L}}\right)$ ) plasma waves $[7,8]$.

In the presence of a dynamical quintessence field weakly coupled to electromagnetism, $\omega_{\sigma} \neq 0$ and the resulting dispersion relations are, according to Eq. (33):

$$
\begin{array}{ll}
\left(n^{2}-\frac{\omega_{\sigma}}{\omega} n-\epsilon_{\mathrm{R}}\right)\left(n^{2}+\frac{\omega_{\sigma}}{\omega} n-\epsilon_{\mathrm{L}}\right)=0, & \theta=0, \\
n^{4}-\left[2 \frac{\epsilon_{\mathrm{L}} \epsilon_{\mathrm{R}}}{\epsilon_{\mathrm{L}}+\epsilon_{\mathrm{R}}}+\epsilon_{\|}+\left(\frac{\omega_{\sigma}}{\omega}\right)^{2}\right] n^{2}+2 \frac{\epsilon_{\|} \epsilon_{\mathrm{L}} \epsilon_{\mathrm{R}}}{\epsilon_{\mathrm{R}}+\epsilon_{\mathrm{L}}}=0, & \theta=\frac{\pi}{2} .
\end{array}
$$

From Eq. (38) the generalized Faraday rotation experienced by the linearly polarized CMB travelling parallel to the magnetic field direction can be obtained as

$$
\Delta \Phi=\frac{\omega}{2 c}\left[\frac{\omega_{\sigma}}{\omega}+\sqrt{\frac{1}{2}\left(\frac{\omega_{\sigma}}{\omega}\right)^{2}+\epsilon_{\mathrm{R}}}-\sqrt{\frac{1}{2}\left(\frac{\omega_{\sigma}}{\omega}\right)^{2}+\epsilon_{\mathrm{L}}}\right] \Delta \mathrm{L},
$$


where $\omega \simeq \omega_{\mathrm{CMB}}$ at decoupling; $\Delta \mathrm{L}$ is the distance travelled by the signal in the direction parallel to the magnetic field direction. It is interesting to compare the contribution of the terms depending upon $\omega_{\sigma}$ and those depending upon the background magnetic field intensity, i.e. the terms appearing in the squared brackets.

According to Eqs. (3),(4) and (15) the leading contribution to the generalized Faraday rotation arises as the sum of two dimensionless ratios, i.e.

$$
\left(\frac{\omega_{\sigma}}{\omega_{\mathrm{CMB}}}\right)+\left(\frac{\omega_{\mathrm{Be}}}{\omega_{\mathrm{CMB}}}\right)\left(\frac{\omega_{\mathrm{pe}}}{\omega_{\mathrm{CMB}}}\right)^{2} .
$$

Using Eqs. (3),(4) and (15), it can be verified directly that the two contributions appearing in Eq. (41) are of the same order, provided

$$
\left(\frac{\beta}{10^{-3}}\right)\left(\frac{M}{M_{\mathrm{P}}}\right) \simeq\left(\frac{B_{0}}{10^{-4} \mathrm{G}}\right)\left(\frac{n_{\mathrm{e}}}{10^{-3} \mathrm{~cm}^{3}}\right),
$$

where in the left-hand-side we computed the quintessential contribution, at the right-handside the contribution of the magnetic field has been reported. The values of $\beta$ and $M$ are, as previously stressed, conservative estimates, compatible with present bounds [5]. For the typical (allowed) parameters reported in Eq. (42), the two contributions then are, as anticipated, of the same order. For the explicit value of $\omega_{\mathrm{CMB}}$ the numerical value $\omega_{\mathrm{CMB}} \sim$ $\mathrm{THz}$ has been used (notice, in fact, that the maximum of the CMB is of the order of the $\mathrm{GHz}$ today and of the order of the $\mathrm{THz}$ around decoupling). Given the possibility of absence of hierarchy between the two dimensionless ratios, Taylor expansions of the obtained disperion relations should be treated with care, especially in view of possible experimental applications of these findings. In a complementary perspective, when analysing the possible rotation of the CMB polarization, it seems then preferable to adopt the generalized formulae derived in the present study.

Concerning the obtained results, a few comments are in order.

- the two contributions have a frequency dependence that may allow us to disentangle their relative weight since the magnetic contribution vanishes for $\omega>\omega_{\mathrm{CMB}}$ as $1 / \omega^{2}$;

- for the set of parameters chosen in Eq. (42) the typical generalized Faraday rotation measurement (i.e. $\Delta \Phi=\mathrm{RM} \lambda_{\mathrm{CMB}}^{2}, \lambda$ being the wavelength) will be of the order of $200 \mathrm{rad} / \mathrm{m}^{2}$ if we use the fact that the optical depth for Thompson scattering at decoupling is $\int x_{\mathrm{e}} n_{\mathrm{e}} a(\eta) d \eta \sim \sigma_{\mathrm{T}}^{-1}$ where $\sigma_{\mathrm{T}}$ is the Thompson cross section;

- situations can be envisaged $\left(\beta \ll 10^{-3}\right)$ where the quintessential contribution is far smaller than the "magnetic" contribution;

- the dispersion relations for radiation propagating orthogonal to the magnetic field intensity also lead, in the case $\omega_{\sigma} \neq 0$, to a rotation where the magnetized contribution is absent and $\Delta \Phi \propto \omega_{\sigma} \Delta \mathrm{L}$; 
- in the (generalized) extraordinary dispersion relations (see Eq. (39) a resonance may appear, when, $\beta \ll 10^{-3}$ and for $\omega_{\mathrm{r}} \simeq \sqrt{\omega_{\mathrm{pe}}^{2}+\omega_{\mathrm{Be}}^{2}}$; however $\omega_{\mathrm{r}} \ll \omega_{\mathrm{CMB}}$;

The derivation presented so far refers to the case of cold magnetoactive plasmas. The effects arising from the finite temperature of the electrons do not modify the leading result obtained in the context of the cold plasma theory. The derivation of the dispersion relations in the case of a warm plasma can be performed, for instance, within a kinetic approach where it can be shown, following the same calculation discussed in the flat space case [12], that the first correction to the leading cold plasma calculation can be recast in an effective redefinition of the plasma frequency for the electrons, namely $\omega_{\mathrm{pe}} \rightarrow \frac{4 \pi n_{0} e^{2}}{m_{e} \gamma}$ where $\gamma=\left(1-\left\langle v^{2}\right\rangle\right)^{-1 / 2}$ and $\left\langle v^{2}\right\rangle$ is the thermal average of the electron velocity.

\section{References}

[1] A. K. Ganguly, S. Konar and P. B. Pal, Phys. Rev. D 60, 105014 (1999); J. C. D'Olivo, J. F. Nieves and S. Sahu, Phys. Rev. D 67, 025018 (2003); A. K. Ganguly and R. Parthasarathy, Phys. Rev. D 68, 106005 (2003).

[2] A. Kosowsky and A. Loeb, Astrophys. J. 469, 1 (1996); M. Giovannini, Phys. Rev. D 56, 3198 (1997).

[3] M. Giovannini, Int. J. Mod. Phys. D 13, 391 (2004); G. Piccinelli and A. Ayala, Lect. Notes Phys. 646, 293 (2004).

[4] J. A. Frieman, C. T. Hill, A. Stebbins and I. Waga, Phys. Rev. Lett. 75, 2077 (1995).

[5] S. M. Carroll, G. B. Field and R. Jackiw, Phys. Rev. D 41, 1231 (1990); S. M. Carroll, Phys. Rev. Lett. 81, 3067 (1998).

[6] K. R. S. Balaji, R. H. Brandenberger and D. A. Easson, JCAP 0312 (2003) 008.

[7] T. J. M Boyd and J. J. Sanderson, The physics of plasmas, (Cambridge University Press, Cambridge, UK, 2003).

[8] H. T. Stix, The theory of plasma waves, (McGraw-Hill, New York, US, 1962).

[9] M. Giovannini, Phys. Rev. D 61, 063004 (2000); Phys. Rev. D 61, 063502 (2000); M. Giovannini and M. E. Shaposhnikov, Phys. Rev. Lett. 80, 22 (1998).

[10] B. Feng, H. Li, M. z. Li and X. m. Zhang, arXiv:hep-ph/0406269.

[11] V. A. Kostelecky, R. Lehnert and M. J. Perry, Phys. Rev. D 68, 123511 (2003); O. Bertolami, R. Lehnert, R. Potting and A. Ribeiro, Phys. Rev. D 69, 083513 (2004).

[12] J. Skilling, Phys. of Fluids 14, 2523 (1971). 